\title{
SOME OBSERVATIONS ON LOCAL AND PROJECTIVE HYPERSURFACES
}

\author{
HAILONG DAO
}

\begin{abstract}
Let $R$ be a hypersurface in an equicharacteristic or unramified regular local ring. For a pair of modules $(M, N)$ over $R$ we study applications of rigidity of $\operatorname{Tor}^{R}(M, N)$, based on ideas by Huneke, Wiegand and Jothilingam. We then focus on the hypersurfaces with isolated singularity and even dimension, and show that modules over such rings behave very much like those over regular local rings. Connections and applications to projective hypersurfaces such as intersections of subvarieties and cohomological criteria for splitting of vector bundles are discussed.
\end{abstract}

\section{Introduction}

The purpose of this note is to continue our investigation of the rigidity and decent intersection of modules over a local hypersurface $R$ done in [Da1]. There we showed that the vanishing of $\theta^{R}(M, N)$, a function introduced by Hochster in [Ho1], implies the rigidity of Tor for $(M, N)$. We will apply this to give various new results on modules over hypersurfaces. We also give supporting evidence for the following Conjecture made in [Da1]:

Conjecture 1.1. Let $R$ be an hypersurface with an isolated singularity. Assume that $\operatorname{dim} R$ is even. Then $\theta^{R}(M, N)$ always vanishes.

This conjecture can be viewed as a consequence of a local version of the following conjecture by Hartshorne on Chow groups of smooth projective hypersurfaces :

Conjecture 1.2. (R.Hartshorne, [Ha1], page 142) Let $X$ be a smooth projective hypersurface in $\mathbb{P}_{\mathbf{C}}^{n}$. Then $\mathrm{CH}^{i}(X)=\mathbb{Z}$ for $i<\operatorname{dim} X / 2$.

Note that the original form of Hartshorne's question states that in codimension less than $\operatorname{dim} X / 2$, a cycle is homologically equivalent to 0 if and only if it is rationally equivalent to 0 . It is not hard to see that Hartshorne's original statement is equivalent to the version stated above. For a K-theoretic discussion of 1.2, see $[\mathrm{Pa}]$ (Conjecture 1.5 and Section 6). For more discussions on how 1.2 is related to 1.1, see [Da1], Section 3. We remark that this connection actually motivated this project, as well as the proof of 4.10 below.

In Section 2 we review the basic notations and preliminary results. In Section 3 we use the procedure invented by Huneke and Wiegand in [HW2] to show that $\operatorname{Hom}_{R}(M, M)$ rarely has good depth. The use of $\theta^{R}(M, N)$ allows us to simplify and strengthen some results in [HW2] (see 3.2 and 3.3, also 4.4 and 4.5).

Section 4 is concerned with two consequences of Conjecture 1.1:

Received by the editors January 31, 2007. 
Conjecture 1.3. Let $R$ be an admissible hypersurface (meaning $\hat{R}$ is a quotient of an unramified or equicharacteristic regular local ring by a nonzero element) with an isolated singularity. Assume that $\operatorname{dim} R$ is even. Let $M, N$ be $R$-modules such that $\operatorname{Tor}_{i}^{R}(M, N)=0$ for some $i$. Then $\operatorname{Tor}_{j}^{R}(M, N)=0$ for all $j \geq i$.

Conjecture 1.4. Let $R$ be an admissible hypersurface with an isolated singularity. Assume that $\operatorname{dim} R$ is even. Let $M, N$ be $R$-modules such that $l\left(M \otimes_{R} N\right)<\infty$. Then $\operatorname{dim} M+\operatorname{dim} N \leq \operatorname{dim} R$.

We will prove 1.1 when $M$ is free on the punctured spectrum of $R$ and $N=M^{*}$ (see 4.1). We also prove 1.4 when $R$ is a standard graded local hypersurface and $M, N$ are graded modules (see 4.11). Many applications follow, such as generalizations of results on $\operatorname{Hom}_{R}(M, M)$ over regular local rings by Auslander and AuslanderGoldman (Corollary 4.8 and 4.9). In that section, the connection with geometry of projective hypersurfaces (which goes both ways) will be exploited. For example, 4.11 will be proved using $l$-adic cohomology, and from 4.1 we obtain the following (see 4.7):

Corollary 1.5. Let $k$ be a field and $n$ an even integer $\geq 4$. Let $X \subset \mathbf{P}_{k}^{n}$ be a nonsingular hypersurface. Let $E$ be a vector bundle on $X$. If $H^{1}\left(X,\left(E \otimes E^{*}\right)(l)\right)=0$ for all $l \in \mathbb{Z}$, then $E$ splits.

In Section 5, we give some further applications of rigidity. The first is a simple characterization of maximal Cohen-Macaulay modules (see 5.1). The second is a connection between vanishing of $\operatorname{Ext}_{R}^{n}(M, M)$ and $\operatorname{pd}_{R} M$, generalizing a result by Jothilingam (see 5.4).

\section{Notation and preliminary results}

Unless otherwise specified, all rings are Noetherian, commutative and local, and all modules are finitely generated. A local ring $(R, m, k)$ is a hypersurface if its completion $\hat{R}$ has the form $T /(f)$, where $T$ is a regular local ring and $f$ is in the maximal ideal of $T$. We say that $R$ is admissible (as a hypersurface) if $T$ is a power series ring over a field or over a discrete valuation ring.

For a ring $R$ and a non-negative integer $i$, we set

$$
X^{i}(R):=\left\{p \in \operatorname{Spec}(R) \mid \operatorname{dim}\left(R_{p}\right) \leq i\right\} .
$$

We denote by $Y(R)$ the set $X^{\operatorname{dim}(R)-1}$, the punctured spectrum of $R$. We say that $R$ satisfy the condition $\left(\mathrm{R}_{i}\right)$ if $R_{p}$ is regular for any $p \in X^{i}(R)$. We denote by $G(R)$ the Grothendieck group of finitely generated modules over $R$ and by $\bar{G}(R):=G(R) /[R]$, the reduced Grothendieck group. Also, we let $\operatorname{Sing}(R):=\{p \in$ $\operatorname{Spec}(R) \mid R_{p}$ is not regular $\}$ be the singular locus of $R$. For an abelian group $G$, we let $G_{\mathbb{Q}}=G \otimes_{\mathbb{Z}} \mathbb{Q}$.

Let $M^{*}:=\operatorname{Hom}(M, R)$ be the dual of $M$. The module $M$ is called reflexive provided the natural map $M \rightarrow M^{* *}$ is an isomorphism. The module $M$ is called maximal Cohen-Macaulay (or sometimes abbreviated as MCM) if $\operatorname{depth}_{R} M=\operatorname{dim} R$.

For a non-negative integer $n, M$ is said to satisfy $\left(\mathrm{S}_{n}\right)$ if :

$$
\operatorname{depth}_{R_{p}} M_{p} \geq \min \left\{n, \operatorname{dim}\left(R_{p}\right)\right\} \forall p \in \operatorname{Spec}(R)
$$

(The depth of the 0 module is set to be $\infty$.). 
A pair of $R$-modules $(M, N)$ is called rigid if for any integer $i \geq 0, \operatorname{Tor}_{i}^{R}(M, N)=0$ implies $\operatorname{Tor}_{j}^{R}(M, N)=0$ for all $j \geq i$. Moreover, $M$ is rigid if for all $N$, the pair $(M, N)$ is rigid.

One defines the finite length index of the pair $(M, N)$ as :

$$
f_{R}(M, N):=\min \left\{i \mid l\left(\operatorname{Tor}_{j}^{R}(M, N)\right)<\infty \text { for } j \geq i\right\}
$$

The function $\theta^{R}(M, N)$

Let $R=T /(f)$ be an admissible local hypersurface. The function $\theta^{R}(M, N)$ was introduced by Hochster ([Ho1]) for any pair of finitely generated modules $M, N$ such that $f_{R}(M, N)<\infty$ as:

$$
\theta^{R}(M, N)=l\left(\operatorname{Tor}_{2 e+2}^{R}(M, N)\right)-l\left(\operatorname{Tor}_{2 e+1}^{R}(M, N)\right) .
$$

where $e$ is any integer $\geq d / 2$. It is well known (see [Ei]) that $\operatorname{Tor}^{R}(M, N)$ is periodic of period at most 2 after $d+1$ spots, so this function is well-defined. The theta function satisfies the following properties (see [Ho1]). First, if $M \otimes_{R} N$ has finite length, then:

$$
\theta^{R}(M, N)=\chi^{T}(M, N) .
$$

Secondly, $\theta^{R}(M, N)$ is biadditive on short exact sequence, assuming it is defined. Specifically, for any short exact sequence:

$$
0 \rightarrow N_{1} \rightarrow N_{2} \rightarrow N_{3} \rightarrow 0
$$

and any module $M$ such that $f_{R}\left(M, N_{i}\right)<\infty$ for all $i=1,2,3$, we have $\theta^{R}\left(M, N_{2}\right)=$ $\theta^{R}\left(M, N_{1}\right)+\theta^{R}\left(M, N_{3}\right)$. Similarly, $\theta(M, N)$ is additive on the first variable.

In [Da1], we show that when $\theta^{R}(M, N)$ can be defined and vanishes, then $(M, N)$ is rigid:

Proposition 2.1. Let $R$ be an admissible hypersurface and $M, N$ be $R$-modules such that $f_{R}(M, N)<\infty$ (so that $\theta^{R}(M, N)$ can be defined). Assume $\theta^{R}(M, N)=0$. Then $(M, N)$ is rigid.

The following corollary will be used frequently in this note:

Corollary 2.2. Let $R$ be an admissible hypersurface and $M, N$ be $R$-modules such that:

(1) $\operatorname{pd}_{R_{p}} M_{p}<\infty$ for any $p \in Y(R)$, the punctured spectrum of $R$ (in particular, this is always true if $R$ has only isolated singularity).

(2) $[N]=0$ in $\bar{G}(R)_{\mathbb{Q}}$.

Then $\theta^{R}(M, N)$ can be defined and equals 0 . Consequently, $(M, N)$ is rigid.

Proof. The condition on $M$ ensures that $\theta^{R}(M, N)$ can be defined for all $R$-module $N$. In other words, $\theta^{R}(M,-)$ gives a $\mathbb{Z}$-linear map from $G(R)$ to $\mathbb{Z}$. The conclusions are now obvious (note that $\theta^{R}(M, R)=0$ ).

\section{The Pushforward}

Let $R$ be a Gorenstein ring and $M$ a torsion-free (equivalent to $\left.\left(\mathrm{S}_{1}\right)\right) R$-module. Consider a short exact sequence :

$$
0 \rightarrow W \rightarrow R^{\lambda} \rightarrow M^{*} \rightarrow 0
$$


Here $\lambda$ is the minimal number of generators for $M^{*}$. Dualizing this short exact sequence and noting that $M$ embeds into $M^{* *}$ we get an exact sequence:

$$
0 \rightarrow M \rightarrow R^{\lambda} \rightarrow M_{1} \rightarrow 0
$$

This exact sequence is called the pushforward of $M$. The following proposition is taken from [HJW].

Proposition 2.3. ([HJW], 1.6) Let $R, M, M_{1}$ as above. Then for any $p \in \operatorname{Spec}(R)$ :

(1) $M_{p}$ is free if and only if $\left(M_{1}\right)_{p}$ is free.

(2) If $M_{p}$ is a maximal Cohen-Macaulay $R_{p}$-module, then so is $\left(M_{1}\right)_{p}$.

(3) $\operatorname{depth}_{R_{p}}\left(M_{1}\right)_{p} \geq \operatorname{depth}_{R_{p}} M_{p}-1$.

(4) If $M$ satisfies $\left(\mathrm{S}_{k}\right)$, then $M_{1}$ satisfies $\left(\mathrm{S}_{k-1}\right)$.

\section{The depth formula}

A result by Huneke and Wiegand showed that when all the high Tor modules vanish, the depths of the modules satisfy a remarkable equation:

Proposition 2.4. ([HW1], 2.5) Let $R$ be a local complete intersection. Let $M, N$ be non-zero finitely generated modules over $R$ such that $\operatorname{Tor}_{i}^{R}(M, N)=0$ for all $i \geq 1$. Then:

$$
\operatorname{depth}(M)+\operatorname{depth}(N)=\operatorname{depth}(R)+\operatorname{depth}\left(M \otimes_{R} N\right)
$$

\section{On depth of $\operatorname{Hom}_{R}(M, M)$}

Throughout this section $R$ is a local hypersurface. We will call an $R$ module $M$ such that $M$ is locally free over the punctured spectrum of $R$ a vector bundle over $Y(R)$ (or just vector bundle). We observe that for a vector bundle $M$ and any $R$-module $N, \theta^{R}(M, N)$ is always defined. In this section we will show that for certain modules over admissible hypersurfaces, $\operatorname{Hom}_{R}(M, M)$ and $M \otimes_{R} M^{*}$ rarely have good depth. We will follow the same procedure as in [HW2], but with two essential additions : we focus on vector bundles from the beginning and we will exploit the function $\theta^{R}(M, N)$ rather heavily. These will allow us to simplify and strengthen some results in [HW2].

Proposition 3.1. Let $R$ be an admissible hypersurface. Let $M$ be a vector bundle over $Y(R)$ such that $\operatorname{depth}(M) \geq 1$. Let $N$ be an $R$-module such that $\theta^{R}(M, N)=0$ and $\operatorname{depth}\left(M \otimes_{R} N\right) \geq 1$. Then $\operatorname{Tor}_{i}^{R}(M, N)=0$ for all $i>0$.

Proof. The assumptions ensure that $M$ is $\mathrm{S}_{1}$. Hence we have the pushforward of $M$ :

$$
0 \rightarrow M \rightarrow R^{\lambda} \rightarrow M_{1} \rightarrow 0
$$

We can tensor with $N$ to get :

$$
0 \rightarrow \operatorname{Tor}_{1}^{R}\left(M_{1}, N\right) \rightarrow M \otimes_{R} N \rightarrow N^{\lambda} \rightarrow M_{1} \otimes_{R} N \rightarrow 0
$$

By 2.3, $M_{1}$ is also a vector bundle. So $l\left(\operatorname{Tor}_{1}^{R}\left(M_{1}, N\right)\right)<\infty$. Since it embeds into a module of positive depth, $\operatorname{Tor}_{1}^{R}\left(M_{1}, N\right)$ must be 0 . Clearly $\theta^{R}\left(M_{1}, N\right)$ is defined and equal to:

$$
\theta^{R}\left(R^{\lambda}, N\right)-\theta^{R}(M, N)=0
$$

So by $2.1, \operatorname{Tor}_{i}^{R}\left(M_{1}, N\right)=0$ for all $i>0$. Since $M \cong \operatorname{syz}_{1}^{R}\left(M_{1}\right)$, we are done. 
The next result is an analogue of Theorem 2.4 in [HW2]

Theorem 3.2. Let $(R, m)$ be an admissible hypersurface of dimension $d$. Let $r$ be an integer such that $0 \leq r<d$. Let $M$ be a vector bundle over $Y(R)$ such that $\operatorname{depth}(M) \geq r$. Let $N$ be an $R$-module satisfying $\left(\mathrm{S}_{r}\right)$, and assume $\theta^{R}(M, N)=0$ and $\mathrm{H}_{m}^{r}\left(M \otimes_{R} N\right)=0$. Then $\operatorname{depth}\left(M \otimes_{R} N\right) \geq r+1$, and if $r>0$ we have $\operatorname{Tor}_{i}^{R}(M, N)=0$ for all $i>0$.

Proof. We will use induction on $r$. If $r=0$ the conclusion is trivial. Now we assume $r>0$. Then $N$ satisfies $\left(\mathrm{S}_{1}\right)$ and we have the pushforward:

$$
0 \rightarrow N \rightarrow R^{\lambda} \rightarrow N_{1} \rightarrow 0
$$

Tensoring with $M$ we get:

$$
0 \rightarrow \operatorname{Tor}_{1}^{R}\left(N_{1}, M\right) \rightarrow N \otimes_{R} M \rightarrow M^{\lambda} \rightarrow N_{1} \otimes_{R} M \rightarrow 0
$$

which we break into two short exact sequences:

$$
0 \rightarrow \operatorname{Tor}_{1}^{R}\left(N_{1}, M\right) \rightarrow N \otimes_{R} M \rightarrow C \rightarrow 0
$$

and:

$$
0 \rightarrow C \rightarrow M^{\lambda} \rightarrow N_{1} \otimes_{R} M \rightarrow 0
$$

Since $M$ is a vector bundle, $T=\operatorname{Tor}_{1}^{R}\left(N_{1}, M\right)$ has finite length. In particular $\mathrm{H}_{m}^{r+1}(T)=0$. By applying $\mathrm{H}_{m}^{0}(-)$ to the first exact sequence we get $\mathrm{H}_{m}^{r}(C)=0$. Now applying $\mathrm{H}_{m}^{0}(-)$ to the second exact sequence and using $\mathrm{H}_{m}^{r-1}(M)=0$ (because $\operatorname{depth}(M) \geq r)$ and $\mathrm{H}_{m}^{r}\left(M \otimes_{R} N\right)=0$ we get $\mathrm{H}_{m}^{r-1}\left(N_{1} \otimes_{R} M\right)=0$. We need to check the other inductive assumptions for $N_{1}$. Clearly, $N_{1}$ is $\left(\mathrm{S}_{r-1}\right)$ by 2.3. Also, $\theta^{R}\left(M, N_{1}\right)$ is defined and equal to:

$$
\theta^{R}\left(M, R^{\lambda}\right)-\theta^{R}(M, N)=0
$$

So by induction we have $\operatorname{depth}\left(M \otimes_{R} N_{1}\right) \geq r$. Then by $3.1 \operatorname{Tor}_{i}^{R}\left(N_{1}, M\right)=0$ for all $i>0$, so we have the last assertion and an exact sequence:

$$
0 \rightarrow N \otimes_{R} M \rightarrow M^{\lambda} \rightarrow N_{1} \otimes_{R} M \rightarrow 0
$$

Therefore $\operatorname{depth}\left(M \otimes_{R} N\right) \geq r$, and since $\mathrm{H}_{m}^{r}\left(M \otimes_{R} N\right)=0$ it follows that $\operatorname{depth}\left(M \otimes_{R}\right.$ $N) \geq r+1$.

Proposition 3.3. Let $R$ be a local hypersurface and $M$ be a vector bundle over $Y(R)$. Assume that $\operatorname{depth}\left(M \otimes_{R} M^{*}\right) \geq 2$ and $\operatorname{Tor}_{i}^{R}\left(M, M^{*}\right)=0$ for all $i>0$. Then $M$ is free.

Proof. By the depth formula we get:

$$
\operatorname{depth}(M)+\operatorname{depth}\left(M^{*}\right) \geq \operatorname{dim} R+2
$$

so by ([Va], 3.3.16) we must have $\operatorname{depth}(M)=\operatorname{depth}\left(M^{*}\right)=\operatorname{dim} R$. On the other hand, the vanishing of all $\operatorname{Tor}_{i}^{R}\left(M, M^{*}\right)$ forces one of the modules to have finite projective dimension (see ([HW2], 1.9). Either way, $M$ must be free (since $M, M^{*}$ are maximal Cohen-Macaulay). 
Theorem 3.4. Let $R$ be an admissible hypersurface satisfying condition $\left(\mathrm{R}_{2}\right)$. Let $M$ be a reflexive $R$-module such that one of the following is satisfied:

1) $\theta^{R}\left(M, M^{*}\right)$ is defined and equals 0 .

1') $[M]=0$ in $\bar{G}(R)_{\mathbb{Q}}$.

If $\operatorname{Hom}_{R}(M, M)$ satisfies $\left(\mathrm{S}_{3}\right)$ then $M$ is free.

Proof. We use induction on $d=\operatorname{dim} R$. If $d \leq 2$ then $R$ is regular, and $M$, being reflexive, must be free. Assume $d \geq 3$ and (by induction hypothesis, since all the conditions localize) that $M$ is free on the punctured spectrum. In other words, $M$ is a vector bundle. Consider the natural map $\phi: M^{*} \otimes_{R} M \rightarrow \operatorname{Hom}(M, M)$. Since $M$ is a vector bundle, the kernel and cokernel of $\phi$ both have finite length. By considering the long exact sequence of local cohomology from the two short exact sequences:

$$
\begin{gathered}
0 \rightarrow \operatorname{ker}(\phi) \rightarrow M^{*} \otimes_{R} M \rightarrow \operatorname{im}(\phi) \rightarrow 0 \\
0 \rightarrow \operatorname{im}(\phi) \rightarrow \operatorname{Hom}(M, M) \rightarrow \operatorname{coker}(\phi) \rightarrow 0
\end{gathered}
$$

and using $\mathrm{H}_{m}^{i}(\operatorname{Hom}(M, M))=0$ for $i<3$ (because $\operatorname{Hom}(M, M)$ is $\left(\mathrm{S}_{3}\right)$ and $d \geq 3$ ) we can deduce that $\mathrm{H}_{m}^{2}\left(M^{*} \otimes_{R} M\right)=0$. Now $M^{*}$ is also a vector bundle, so $\theta^{R}\left(-, M^{*}\right)$ is always defined. Therefore if $[M]=0$ in $\bar{G}(R)_{\mathbb{Q}}$ then $\theta^{R}\left(M, M^{*}\right)=0$. So in both cases 1) and $\left.1^{\prime}\right), \theta^{R}\left(M, M^{*}\right)=0$ and Theorem $3.2 \operatorname{implies} \operatorname{depth}\left(M \otimes_{R} M^{*}\right) \geq 3$ as well as $\operatorname{Tor}_{i}^{R}\left(M, M^{*}\right)=0$ for all $i>0$. The result now follows from Proposition 3.3 .

Example 3.5. This will be our main example throughout this note. Let $k$ be a field and $R=k[[x, y, u, v]] /(x u-y v)$ with $m=(x, y, u, v)$ and $M=(x, y)$. We claim that $M^{*} \cong(x, v)$. Any $R$-linear map $\phi$ from $M$ to $R$ is determined by $\phi(x)$. Hence $M^{*}$ is isomorphic to $\{a \in R \mid y a \in x R\}$, which is easily seen to be $(x, v)$. So $M \otimes_{R} M^{*} \cong$ $\left(x^{2}, x y, x v, y v\right) \cong\left(x^{2}, x y, x v, x u\right) \cong m$ (see Example 1.8 of [HW2]). Using the long exact sequence of local cohomology for the sequence $0 \rightarrow m \rightarrow R \rightarrow k \rightarrow 0$ we get $\mathrm{H}_{m}^{1}\left(M \otimes_{R} M^{*}\right)=\mathrm{H}_{m}^{1}(m)=k$ and $\mathrm{H}_{m}^{2}\left(M \otimes_{R} M^{*}\right)=\mathrm{H}_{m}^{2}(m)=0$. Clearly $\operatorname{depth}\left(M \otimes_{R} M^{*}\right)=\operatorname{depth}(m)=1$. Obviously, $M$ is not free.

Note that both $M$ and $M^{*}$ are maximal Cohen-Macaulay modules over $R$. Since $R$ has an isolated singularity, they are also vector bundles over $Y(R)$. It can be easily computed that $\operatorname{Tor}_{1}^{R}\left(M, M^{*}\right)=k$, $\operatorname{Tor}_{2}^{R}\left(M, M^{*}\right)=0$ and $\theta^{R}\left(M, M^{*}\right)=-1$.

Consider Proposition 3.1 and Theorem 3.2. Let $N=M^{*}$. Then the example shows that the condition $\theta^{R}(M, N)=0$ can not be dropped.

Consider Theorem 3.4. Note that $\bar{G}(R)_{\mathbb{Q}}=\mathbb{Q}[M] \cong \mathbb{Q}$. The example shows that the condition $M=0$ in $\bar{G}(R)_{\mathbb{Q}}$ can not be dropped either.

\section{Isolated hypersurface singularities of even dimensions}

In this section we will show some supporting evidence for Conjecture 1.1. Our results indicate that modules over isolated hypersurface singularities of even dimensions behave very similarly to those over regular local rings. We first prove:

Theorem 4.1. Let $R$ be a hypersurface with isolated singularity. Assume that $\operatorname{dim} R$ is even. Then for any vector bundle $M$ on $Y(R), \theta^{R}\left(M, M^{*}\right)=0$. 
We need to review the concept of stable (co)homology (for more details, see [Bu] or $[\mathrm{AB}])$. Let $R$ be a Noetherian ring, a complete resolution of an $R$-module $M$ is a complex $\mathbf{T}$ such that $\mathrm{H}_{n}(\mathbf{T})=\mathrm{H}^{n}\left(\mathbf{T}^{*}\right)=0$ for all $n \in \mathbb{Z}$ and $\mathbf{T}_{\geq r}=\mathbf{P}_{\geq r}$ for some projective resolution $\mathbf{P}$ of $M$ and some integer $r$. It is known that the modules $\mathrm{H}^{i}\left(\operatorname{Hom}_{R}(\mathbf{T}, N)\right)$ and $\mathrm{H}_{i}\left(\mathbf{T} \otimes_{R} N\right)$ are independent of the resolution, and one calls them $\widehat{\operatorname{Ext}}_{R}^{i}(M, N)$ and $\widehat{\operatorname{Tor}}_{i}^{R}(M, N)$, respectively.

Before moving on we recall the Local Duality Theorem in our context and some consequences that will be used. Let $d=\operatorname{dim} R$. Let ${ }^{\vee}$ denote $\operatorname{Hom}_{R}(-, E(k))$, the Matlis dual. Then for any module $M$ we have an isomorphism:

$$
\operatorname{Ext}_{R}^{i}(M, R) \cong \mathrm{H}_{m}^{d-i}(M)^{\vee}
$$

In particular, if $M$ is maximal Cohen-Macaulay, then $\operatorname{Ext}_{R}^{i}(M, R)=0$ for $i>0$ and if $l(M)<\infty$ then $\operatorname{Ext}_{R}^{i}(M, R)=0$ for $i \neq d$. Also, if $M$ is maximal CohenMacaulay then so is $M^{*}$, since by dualizing a free resolution of $M$ one can see that $M^{*}$ is a syzygy of infinitely high order.

We will need the standard and easy results below, reproved for the reader's convenience (some of these could be found in $[\mathrm{AB}]$, but we could not find a full reference):

Lemma 4.2. Let $R$ be a local hypersurface. Let $M, N$ be $R$-modules such that $M$ is maximal Cohen-Macaulay (MCM).Then we have the following isomorphism:

(1) $\operatorname{Tor}_{i}^{R}(M, N) \cong \widehat{\operatorname{Tor}}_{i}^{R}(M, N)$ for all $i>0$.

(2) $\operatorname{Ext}_{R}^{i}(M, N) \cong \widehat{\operatorname{Ext}}_{R}^{i}(M, N)$ for all $i>0$.

(3) $\widehat{\operatorname{Tor}}_{i}^{R}(M, N) \cong \widehat{\operatorname{Tor}}_{i+2}^{R}(M, N)$ for all $i$.

(4) $\widehat{\operatorname{Ext}}_{R}^{i}(M, N) \cong \widehat{\operatorname{Ext}}_{R}^{i+2}(M, N)$ for all $i$.

(5) $\widehat{\operatorname{Tor}}_{i}^{R}(M, N) \cong \widehat{\operatorname{Ext}}_{R}^{-i-1}\left(M^{*}, N\right)$ for all $i$.

(6) $\operatorname{Tor}_{i}^{R}(M, N) \cong \widehat{\operatorname{Ext}}_{R}^{i+1}\left(M^{*}, N\right)$ for all $i>0$.

Proof. Let $\mathbf{F}: \ldots \rightarrow F_{1} \rightarrow F_{0} \rightarrow M^{*} \rightarrow 0$ be a free resolution of $M^{*}$. Since $M$ is MCM we have $M^{* *}=M$ and $\operatorname{Ext}^{n}\left(M^{*}, R\right)=0$ for all $n>0$. So dualizing $\mathbf{F}$ we get an exact sequence:

$$
\mathbf{F}^{*}: \quad 0 \rightarrow M \rightarrow F_{0}^{*} \rightarrow F_{1}^{*} \rightarrow \ldots
$$

Now let $\mathbf{G}: \ldots \rightarrow G_{1} \rightarrow G_{0} \rightarrow M \rightarrow 0$ be a minimal free resolution of $M$. We can splice $\mathbf{G}$ and $\mathbf{F}^{*}$ together to get an exact sequence:

$$
\mathbf{T}: \ldots \rightarrow G_{1} \rightarrow G_{0} \rightarrow F_{0}^{*} \rightarrow F_{1}^{*} \rightarrow \ldots
$$

It is obvious that $\mathbf{T}$ is a complete resolution of $M$. That proves (1) and (2). Since $M, M^{*}$ are MCM and $R$ is a hypersurface, $\mathbf{G}, \mathbf{F}$ are periodic of period at most 2 . In particular, $\operatorname{syz}_{2 n}^{R} M^{*} \cong M^{*}$ for any $n>0$. Let $M_{i}=\operatorname{im}\left(F_{i-1}^{*} \rightarrow F_{i}^{*}\right)$. Then $M_{2 n} \cong\left(\operatorname{syz}_{2 n}^{R} M^{*}\right)^{*} \cong M^{* *} \cong M$. Thus for any $n>0$ :

$$
\ldots \rightarrow G_{1} \rightarrow G_{0} \rightarrow F_{0}^{*} \rightarrow F_{1}^{*} \rightarrow \ldots \rightarrow F_{2 n-1}^{*} \rightarrow 0
$$

is a free resolution of $M$. Hence (3) and (4) follow. From the construction, $\mathbf{T}^{*}[-1]$ is a complete resolution of $M^{*}$, and the canonical isomorphism $\operatorname{Hom}_{R}\left(\mathbf{T}^{*}, N\right) \cong\left(\mathbf{T} \otimes_{R} N\right)$ gives (5). Finally, (6) follows from combining (1), (5) and (4).

We will also need the following result by Buchweitz: 
Proposition 4.3. ([Bu],10.3.3) Let $R$ be a local hypersurface with isolated singularity such that $d=\operatorname{dim} R$ is even. Then for any two MCM R-modules $M, N$, and integers $i, j$ such that $i-j$ is odd:

$$
l\left(\widehat{\operatorname{Ext}}_{R}^{i}(M, N)\right)=l\left(\widehat{\operatorname{Ext}}_{R}^{j}\left(M^{*}, N^{*}\right)\right)
$$

Proof. Since $[\mathrm{Bu}]$ is not available publicly, and in any case the assertion was derived there from very general theory, we will summarize a self-contained proof. Let $T$ be the complete resolution of $M$ constructed in the proof of 4.2 and $I$ be an (finite) injective resolution of $R$ over $R$. One can see that the total complexes associated to the double complexes $\operatorname{Hom}_{R}\left(\operatorname{Hom}_{R}(T, N), I\right)$ and $\operatorname{Hom}_{R}\left(T^{*}, \operatorname{Hom}(N, I)\right)$ are isomorphic. Note that $T^{*}[-1]$ is a complete resolution of $M^{*}$. We get two spectral sequences converging to the same limit:

$$
{ }^{1} E_{2}^{i, j}=\operatorname{Ext}_{R}^{i}\left(\widehat{\operatorname{Ext}}_{R}^{-j}(M, N), R\right) \Rightarrow H^{i+j}
$$

and

$$
{ }^{2} E_{2}^{i, j}=\widehat{\operatorname{Ext}}_{R}^{i-1}\left(M^{*}, \operatorname{Ext}_{R}^{j}(N, R) \Rightarrow H^{i+j}\right.
$$

As $N$ is MCM, $\operatorname{Ext}_{R}^{j}(N, R)=0$ for $j>0$, so the second sequence collapses, leaving $H^{i+j} \cong \widehat{\operatorname{Ext}}_{R}^{i+j-1}\left(M^{*}, N^{*}\right)$. On the other hand, $M$ is free on the punctured spectrum, so $l\left(\widehat{\operatorname{Ext}}_{R}^{-j}(M, N)\right)$ is finite. Thus ${ }^{1} E_{2}^{i, j}=0$ unless $i=d$, which gives $H^{d+j} \cong$ $\operatorname{Ext}_{R}^{d}\left(\widehat{\operatorname{Ext}}_{R}^{-j}(M, N), R\right) \cong \widehat{\operatorname{Ext}}_{R}^{-j}(M, N)^{\vee}$. Putting everything together we get:

$$
\widehat{\operatorname{Ext}}_{R}^{-j}(M, N)^{\vee} \cong \widehat{\operatorname{Ext}}_{R}^{d+j-1}\left(M^{*}, N^{*}\right)
$$

Since $d$ is even and taking Matlis dual preserves length, part (4) of 4.2 finishes our proof.

Now we can prove 4.1:

Proof. (of Theorem 4.1) First, we will prove the theorem for the case when $M$ is MCM. In this situation, for any integer $i>0$, by (6) of 4.2 we have:

$$
\operatorname{Tor}_{i}^{R}\left(M, M^{*}\right) \cong \widehat{\operatorname{Ext}}_{R}^{i+1}\left(M^{*}, M^{*}\right)
$$

and

$$
\operatorname{Tor}_{i+1}^{R}\left(M^{*}, M\right) \cong \widehat{\operatorname{Ext}}_{R}^{i+2}(M, M)
$$

Hence 4.3 (applied for $N=M$ ) shows that $\theta^{R}\left(M, M^{*}\right)=0$.

Now, assume $M$ is a vector bundle. Let $K=\operatorname{syz}_{1}^{R}(M)$. We want to prove that $\theta^{R}\left(M, M^{*}\right)=\theta^{R}\left(K, K^{*}\right)$. Dualizing the short exact sequence: $0 \rightarrow K \rightarrow F \rightarrow M \rightarrow$ 0 we get an exact sequence:

$$
0 \rightarrow M^{*} \rightarrow F^{*} \rightarrow K^{*} \rightarrow \operatorname{Ext}_{R}^{1}(M, R) \rightarrow 0
$$

So $M^{*}+K^{*}=\operatorname{Ext}_{R}^{1}(M, R)$ in $\bar{G}(R)$. Note that $\operatorname{Ext}_{R}^{1}(M, R)$ has finite length because $M$ is free on the punctured spectrum of $R$. We claim that any module of finite length is equal to 0 in $\bar{G}(R)_{\mathbb{Q}}$. Since any module of finite length is a multiple of $[k]$, it is enough to prove the claim for one finite length module. If $\operatorname{dim} R=0$ then there is nothing to prove. If $\operatorname{dim} R>0$ then pick a prime $p$ such that $\operatorname{dim} R / p=1$ and a non-unit $x \notin p$. The exact sequence $0 \rightarrow R / p \rightarrow R / p \rightarrow R /(p+x) \rightarrow 0$ shows that $[R /(p+x)]=0$, which is all we need. So $\left[M^{*}\right]=-\left[K^{*}\right]$ and $\theta^{R}\left(M^{*},-\right)=-\theta^{R}\left(K^{*},-\right)$. Since $\theta^{R}(M,-)=-\theta^{R}(K,-)$, we have $\theta^{R}\left(M, M^{*}\right)=\theta^{R}\left(K, K^{*}\right)$. 
Repeating the equality above we get $\theta^{R}\left(M, M^{*}\right)=\theta^{R}\left(K, K^{*}\right)$ when $K=\operatorname{syz}_{n}^{R}(M)$ for any $n>0$. But for $n \gg 0 K$ is an MCM $R$-module, so $\theta^{R}\left(K, K^{*}\right)=0$.

Corollary 4.4. Let $R$ be an admissible hypersurface such that $R$ has an isolated singularity and $\operatorname{dim} R$ is an even number greater than 2 . Let $M$ be a reflexive $R$ module. If $\operatorname{Hom}_{R}(M, M)$ satisfies $\left(\mathrm{S}_{3}\right)$ then $M$ is free.

Proof. By localizing on $p \in Y(R)$ and using Theorem 3.4 (note that a regular local ring can also be considered a hypersurface) we may assume that $M$ is a vector bundle. The result then follows from 3.4 and 4.1 .

Corollary 4.5. Let $R$ be an admissible hypersurface such that $R$ has isolated singularity and $\operatorname{dim} R>2$ and is even. Let $M$ be a vector bundle over $Y(R)$. Assume that $M$ is reflexive and $\mathrm{H}_{m}^{2}\left(M \otimes_{R} M^{*}\right)=0$. Then $M$ is free.

Proof. By 3.2 and 4.1 we have $\operatorname{Tor}_{i}^{R}\left(M, M^{*}\right)=0$ for all $i>0$. Thus Proposition 3.3 shows that $M$ is free.

Example 4.6. Let $R, M$ as in Example 3.5. Then $\mathrm{H}_{m}^{2}\left(M \otimes_{R} M^{*}\right)=0$ but $M^{*}$ is not free. Note that $\operatorname{dim} R=3$.

Corollary 4.7. Let $k$ be a field and $n$ an even integer $\geq 4$. Let $X \subset \mathbf{P}_{k}^{n}$ be a nonsingular hypersurface. Let $E$ be a vector bundle on $X$. If $H^{1}\left(X,\left(E \otimes E^{*}\right)(l)\right)=0$ for all $l \in \mathbb{Z}$, then $E$ splits.

Proof. This is a straightforward consequence of standard connections between a projective variety and its affine cone (see section 5 in [HW2]). Let $A$ be the homogenous coordinate ring of $X, m$ be the irrelevant ideal and $R=A_{m}$. Let $N=$ $\oplus_{i \in \mathbb{Z}} H^{0}(X, E(i))$ and $M=N_{m}$. Note that the cohomology condition on $E$ translates to: $\mathrm{H}_{m}^{2}\left(M \otimes_{R} M^{*}\right)=0$. Now we can apply 4.5 to conclude that $M$ is a free $R$-module, which means $E$ splits.

It is worth noting two obvious consequences of Corollary 4.4 which are generalizations of well-known results by Auslander and Goldman ([AG], Theorem 4.4) and Auslander ([Au], Theorem 1.3) for modules over regular local rings:

Corollary 4.8. Let $R$ be an admissible hypersurface such that $R$ has isolated singularity and $\operatorname{dim} R>2$ and is even. Let $M$ be a reflexive $R$-module. If $\operatorname{Hom}_{R}(M, M) \cong R^{n}$ for some $n$ then $M$ is free.

Corollary 4.9. Let $R$ be an admissible hypersurface such that $R$ has isolated singularity and $\operatorname{dim} R>2$ and is even. Let $M$ be an $R$-module satisfying $\left(\mathrm{S}_{3}\right)$. If $\operatorname{Hom}_{R}(M, M) \cong M^{n}$ for some $n$ then $M$ is free.

We now show that Conjecture 1.4 is true in the standard graded case:

Theorem 4.10. Let $k$ be an algebraically closed field and $n$ an odd integer. Let $X \subset \mathbf{P}_{k}^{n+1}$ be a smooth projective hypersurface. Let $U, V$ be subvarieties of $X$ such that $U \cap V=\emptyset$. Then $\operatorname{dim} U+\operatorname{dim} V<\operatorname{dim} X$. 
Proof. We are going to use $l$-adic cohomology (for basic properties and notations, we refer to $[\mathrm{Mi}]$ or $[\mathrm{Sha}])$. Let $l$ be a prime number such that $l \neq \operatorname{char}(k)$. There is a class map:

$$
c l: \mathrm{CH}^{r}(X) \rightarrow H^{2 r}\left(X, \mathbb{Q}_{l}(r)\right)
$$

This map gives a graded rings homeomorphism $\mathrm{CH}^{*}(X) \rightarrow \oplus H^{2 r}\left(X, \mathbb{Q}_{l}(r)\right.$ ) (with the intersection product on the left hand side and the cup product on the right hand side, see [Mi], VI, 10.7 and 10.8). Let $a=\operatorname{dim} U$ and $b=\operatorname{dim} V$, and we may assume $a \geq b$. Suppose $a+b=\operatorname{dim} X=n$ (if $a+b>n$, we can always choose some subvariety of smaller dimension inside $U$ or $V$ such that equality occurs). Then $2 a \geq n$, but $n$ is odd, so $2 a>n$. Let $h \in \mathrm{CH}^{1}(X)$ represent the hyperplane section. By Weak Lefschetz Theorem (see, for example, [Sha], 7.7, page 112) and the fact that $2(n-a)<n$, we have:

$$
H^{2(n-a)}\left(X, \mathbb{Q}_{l}(n-a)\right) \cong H^{2(n-a)}\left(\mathbf{P}_{k}^{N}, \mathbb{Q}_{l}(n-a)\right)
$$

The latter is generated by a power of the class of the hyperplane section. Thus $c l(U)=c l(h)^{n-a}$ in $H^{2(n-a)}\left(X, \mathbb{Q}_{l}(n-a)\right)$. We then have :

$$
\operatorname{cl}(U . V)=\operatorname{cl}(U) \cup \operatorname{cl}(V)=\operatorname{cl}(h)^{n-a} \cup \operatorname{cl}(V)=\operatorname{cl}\left(h^{n-a} . V\right)
$$

The last term is equal to the degree of $h^{n-a} . V \in \mathrm{CH}^{n}(X)$, so it is nonzero. But the first term has to be 0 by assumption. This contradiction proves the Theorem.

Corollary 4.11. Let $k$ be a perfect field and $n$ an even integer. Suppose that $A=$ $k\left[x_{0}, \ldots, x_{n}\right] /(F)$ is a homogenous hypersurface. Let $(R, m, k)$ be the local ring at the origin of $A$. Suppose that $R$ has an isolated singularity. Let $M, N$ be graded $R$-modules such that $l\left(M \otimes_{R} N\right)<\infty$. Then $\operatorname{dim} M+\operatorname{dim} N \leq \operatorname{dim} R$.

Proof. Without affecting matters we may assume $k$ is algebraically closed (it would not affect the isolated singularity condition because $k$ is perfect, so we can compute the singular locus of $A$ by the Jacobian ideal, which would not be changed by extending $k$ to its algebraic closure). Since the minimal primes of a graded modules are homogenous, we may replace $M$ and $N$ with $R / P$ and $R / Q$, where $P, Q$ are homogenous primes in $R$. Now let $X=\operatorname{Proj}(A), U=\operatorname{Proj}(A / P), V=\operatorname{Proj}(A / Q)$ and applying the previous Theorem.

\section{Some other applications}

In this section we discuss some further applications of rigidity. The theme is that some strong conditions on a module could be detected by the vanishing of a single Ext or Tor module. We first note a simple characterization of maximal Cohen-Macaulay modules, due to the fact that over an admissible hypersurface, a module of finite length is rigid (see 2.4 in [HW1] or 4.10 in [Da1]):

Proposition 5.1. Let $R$ be a admissible hypersurface and $M$ an $R$-module. The following are equivalent:

(1) There is a nonzero finite length module $N$ such that $\operatorname{Tor}_{1}^{R}(M, N)=0$.

(2) There is a nonzero finite length module $N$ such that $\operatorname{Ext}_{R}^{1}(M, N)=0$.

(3) $M$ is a maximal Cohen-Macaulay R-module. 
Proof. For equivalence of (1) and (2): we may assume $R$ is complete. Then we have a well-known isomorphism (see, for example, page 7 of $[\mathrm{EG}]$ ) $\operatorname{Tor}_{1}^{R}(M, N)^{\vee} \cong$ $\operatorname{Ext}_{R}^{1}\left(M, N^{\vee}\right)$, so (1) and (2) are equivalent.

Assume (1). Then since $N$ is rigid, we have $\operatorname{Tor}_{i}^{R}(M, N)=0$ for all $i \geq 1$. The depth formula shows:

$$
\operatorname{depth}(M)+\operatorname{depth}(N)=\operatorname{depth}(R)+\operatorname{depth}\left(M \otimes_{R} N\right)
$$

hence $\operatorname{depth}(M)=\operatorname{depth}(R)$ and $(3)$ follows.

Finally, assume (3). Let $n=\operatorname{dim} R$, we can choose a regular sequence $x_{1}, \ldots, x_{n}$ on both $M$ and $R$ (by induction on $n$ ). Now, just take $N=R /\left(x_{1}, \ldots, x_{n}\right)$. Then $N$ has finite length and $\operatorname{Tor}_{1}^{R}(M, N)=0$.

It would be very interesting to know whether the previous Proposition is true for all local complete intersections. It is not hard to see that this question has intimate connection to the rigidity of modules of finite length over such rings:

Lemma 5.2. Let $\mathcal{C}$ be the category of all local complete intersections. Consider the following properties:

(1) For any $R \in \mathcal{C}$, any $R$-module of finite length is rigid.

(2) For any $R \in \mathcal{C}$ and any $R$-module $M$, if there is a nonzero finite length $R$-module $N$ such that $\operatorname{Tor}_{1}^{R}(M, N)=0$ then $M$ is maximal Cohen-Macaulay.

(3) For any $R \in \mathcal{C}$ such that $\operatorname{dim} R>0$ and any $R$-module $M$, if there is a nonzero finite length $R$-module $N$ such that $\operatorname{Tor}_{1}^{R}(M, N)=0$ then $\operatorname{depth}(M)>0$.

(4) For any $R \in \mathcal{C}$, any $R$-module of finite length and finite projective dimension is rigid.

We have $(1) \Rightarrow(2) \Leftrightarrow(3) \Rightarrow(4)$.

Proof. $(1) \Rightarrow(2)$ is the proof of 5.1 .

$(2) \Leftrightarrow(3)$. (2) clearly implies (3). Suppose (3) holds, and let $M$ as in (2). We may assume $\operatorname{dim} R>1$. Then $\operatorname{since} \operatorname{depth}(M), \operatorname{depth}(R)>0$ we can find $x \in \operatorname{Ann}_{R} N$ such that $x$ is regular on both $R$ and $M$. Then $\operatorname{Tor}_{1}^{R /(x)}(M / x M, N) \cong \operatorname{Tor}_{1}^{R}(M, N)=0$. So we can replace $R, M$ by $R /(x), M / x M$ and repeat the process until $\operatorname{dim} R=1$, at which point $M$ is MCM by (3).

$(2) \Rightarrow(4)$. Suppose $M, N$ are nonzero $R$-modules such that $l(N)<\infty, \operatorname{pd}_{R} N<$ $\infty$ and $\operatorname{Tor}_{i}^{R}(M, N)=0$ for some $i>0$. Then let $M^{\prime}=\operatorname{syz}_{i-1}^{R} M$ and we get $\operatorname{Tor}_{1}^{R}\left(M^{\prime}, N\right)=0$. By (2) $M^{\prime}$ is MCM. Let $q$ be the largest integer such that $\operatorname{Tor}_{q}^{R}\left(M^{\prime}, N\right) \neq 0$. Then by Lemma 2.2 in [HW2] we have the following equality (since $\left.\operatorname{depth}\left(\operatorname{Tor}_{q}^{R}\left(M^{\prime}, N\right)\right)=0\right)$ :

$$
\operatorname{depth}\left(M^{\prime}\right)+\operatorname{depth}(N)=\operatorname{depth}(R)-q
$$

Since $\operatorname{depth}\left(M^{\prime}\right)=\operatorname{depth}(R)$ and $\operatorname{depth}(N)=0$ we must have $q=0$.

Remark. In [JS] a module $M$ is constructed over a 0-dimensional Gorenstein ring such that $M$ is not rigid. So property (1) fails for Gorenstein rings.

Finally, we want to use our approach to rigidity to prove a connection between vanishing of Ext and projective dimension, generalizing a result by Jothilingam. In [Jot], the following was proved: 
Theorem 5.3. ([Jot], Theorem) Let $R$ be a regular local ring and $M$ an $R$-module. Then $\operatorname{Ext}_{R}^{n}(M, M)=0$ if and only if $\operatorname{pd}_{R}(M)<n$.

In [Jo], Jorgensen observed that Jothilingam's result is true for any local ring $R$ if we assume that $M$ is rigid. We will modify Jothilingam's proof to show:

Proposition 5.4. Let $R$ be an admissible hypersurface and $M$ be an $R$-module such that $M=0$ in $\bar{G}(R)_{\mathbb{Q}}$. Then $\operatorname{Ext}_{R}^{n}(M, M)=0$ if and only if $\operatorname{pd}_{R}(M)<n$.

Remark. Over a hypersurface, the classes of rigid modules and of modules which are 0 in $\bar{G}(R)_{\mathbb{Q}}$ are incomparable (see [Da1]).

Proof. We first need to review some notation in [Jot]. Let $N$ be an $R$-module and let

$$
F=\ldots \rightarrow F_{2} \rightarrow F_{1} \rightarrow F_{0} \rightarrow N \rightarrow 0
$$

be a minimal free resolution of $N$. Then one can define for $i \geq 0$ :

$$
D^{i}(N)=\operatorname{coker}\left(F_{i}^{*} \rightarrow F_{i+1}^{*}\right)
$$

Note that $D^{i}(N)$ can be computed, up to free summand, from any resolution of $N$. We will use induction on $\operatorname{dim} R$. The proof of the Theorem in [Jot] shows that to prove $\operatorname{Ext}_{R}^{n}(M, M)=0$ implies $\operatorname{pd}_{R}(M)<n$ one only needs that the pair $\left(D^{n}(M), M\right)$ is rigid. Let $L=D^{n}(M)$. We will show that $\theta^{R}(L, M)=0$. If $\operatorname{dim} R=0$ then $\theta^{R}(-,-)$ is always defined and equals 0 , since all modules have finite length and $\bar{G}(R)_{\mathbb{Q}}=0$. Take any prime $p \in Y(R)$, the punctured spectrum. By induction hypothesis $\left(\operatorname{syz}_{n-1}^{R} M\right)_{p}$ is free, so $L_{p}$ is a free $R_{p^{-}}$module. Thus $\theta^{R}(L,-)$ is always defined and since $M=0$ in $\bar{G}(R)_{\mathbb{Q}}$ we must have $\theta^{R}(L, M)=0$. So $(L, M)$ is rigid by 2.1 and we are done.

Example 5.5. Let $R=k[[x, y]] /(x y)$ and $M=R /(x)$. Then $\operatorname{Ext}^{2 i+1}(M, M)=0$ for all $i>0$ but $\operatorname{pd}_{R} M=\infty$. Note that $\bar{G}(R) \cong \mathbb{Z}[M] \cong \mathbb{Z}$.

\section{Ackowledgements}

We would like to thank Ragnar-Olaf Buchweitz, David Jorgensen, Craig Huneke, Mircea Mustaţă, Paul Roberts, Vasudevan Srinivas and Claire Voisin for many useful conversations and communications regarding this note. We also thank the anonymous referee whose reports have tremendously improved the presentation and mathematical content of the note.

\section{References}

[Au] M. Auslander, On the Purity of the Branch Locus, Amer. J. Math. 84 (1962), 116-125.

[AB] L. L. Avramov and R.-O. Buchweitz, Support varieties and cohomology over complete intersections, Invent. Math. 142 (2000), 285-318.

[AG] M. Auslander and O. Goldman, Maximal Orders, Trans. Amer. Math. Soc. 97 (1960), 1-24.

[BH] W. Bruns and J. Herzog, Cohen-Macaulay rings, Cambridge Univ. Press, Cambridge (1996).

[Bu] R.-O. Buchweitz, Maximal Cohen-Macaulay modules and Tate cohomology over Gorenstein rings, preprint, Unive. Hannover, (1986).

[Da1] H. Dao, Decency and rigidity over hypersurfaces, arXiv math.AC/0611568, preprint.

[EG] E. G. Evans and P. Griffith, Sygyzies, Lond. Math. Soc. Lect. Notes 106 (1985).

[Ei] D. Eisenbud, Homological algebra on a complete intersection, with an application to group representations, Tran. Amer. Math. Soc. 260 (1980), 35-64. 
[Fu] W. Fulton, Intersection Theory, Springer-Verlag, Berlin (1998).

[GD] A. Grothendieck and J. Dieudonné, Eléments de géometrique algébrique, IV Publ. Math. I.H.E.S. 24 (1965).

[Ha1] R. Hartshorne, Equivalence relations on algebraic cycles and subvarieties of small codimension, Proc. Symp. Pure Math. 29 (1975), 129-164.

[Ha2] Algebraic Geometry, Graduate Text in Mathematics, Springer-Verlag, New York, (1977).

[Ho1] M. Hochster, The dimension of an intersection in an ambient hypersurface, Proceedings of the First Midwest Algebraic Geometry Seminar (Chicago Circle,1980), Lecture Notes in Mathematics 862, Springer-Verlag, 1981, 93-106.

[HW1] C. Huneke and R. Wiegand, Tensor products of modules and the rigidity of Tor, Math. Ann. 299 (1994), 449-476.

[HW2] - Tensor products of modules, rigidity and local cohomology, Math. Scan. 81 (1997), $161-183$.

[HJW] C. Huneke, R. Wiegand, and D. Jorgensen, Vanishing theorems for complete intersections, J. Algebra 238 (2001), 684-702.

[Jo] D. Jorgensen, Finite projective dimension and the vanishing of $\operatorname{Ext}(M, M)$, preprint.

[Jot] P. Jothilingam, A note on grade, Nagoya Math. J. 59 (1975), 149-152.

[JS] D. Jorgensen and L. Sega, Nonvanishing cohomology and classes of Gorenstein rings, Adv. Math. 188 (2004), 470-490.

[Mi] J. S. Milne, Etale cohomology, Princeton Univ. Press (1980)

[Pa] K. H. Paranjape, Cohomological and cycle-theoretic connectivity, Ann. of Math. 140 (1994), 641-660.

[PS] C. Peskine and L. Szpiro, Dimension projective finie et cohomologie locale. Applications à la démonstration de conjectures de M. Auslander, H. Bass et A. Grothendieck, Inst. Hautes tudes Sci. Publ. Math. 42 (1973), 47-119.

[Ro] P. Roberts, Multiplicities and Chern classes in Local Algebra, Cambridge Univ. Press, Cambridge (1998).

[Sha] I. R. Shafarevich (Ed.), Algebraic Geometry II. Cohomology of algebraic varieties. Algebraic surfaces, Encyclopaedia of Mathematical Sciences, 35, Springer, Berlin (1995).

[Va] W. Vasconcelos, Arithmetic of Blowup Algebra, London Math. Soc. Lecture Notes 195 (1994).

Department of Mathematics, University of Utah, 155 South 1400 East, Salt Lake City, UT 84112-0090, USA

E-mail address: hdao@math.utah.edu 